\title{
Diffuse Interstellar Bands in (Proto-) Fullerene-Rich Environments
}

\author{
D. A. García-Hernández ${ }^{1,2}$ \\ ${ }^{1}$ Instituto de Astrofísica de Canarias, C/ Via Láctea s/n, E-38200 La Laguna, Tenerife, Spain \\ email: agarcia@iac.es \\ ${ }^{2}$ Departamento de Astrofísica, Universidad de La Laguna (ULL), E-38206 La Laguna, \\ Tenerife, Spain
}

\begin{abstract}
The recent infrared detection of fullerenes $\left(\mathrm{C}_{60}\right.$ and $\left.\mathrm{C}_{70}\right)$ in Planetary Nebulae (PNe) and R Coronae Borealis (RCB) stars offers a beautiful opportunity for studying the diffuse interstellar bands (DIBs) in sources where fullerenes are abundant. Here we present for the first time a detailed inspection of the optical spectra of the hot RCB star DY Cen and two fullerene PNe (Tc 1 and M 1-20), which permits us to directly explore the fullerenes - DIB connection. The DIB spectrum of DY Cen (García-Hernández et al. 2012a) is remarkably different from that in fullerene PNe (García-Hernández \& Díaz-Luis 2013). In particular, Tc 1 displays unusually strong $4428 \AA$ and $6309 \AA$ DIBs, which are normal (or not seen) in DY Cen. On the other hand, DY Cen displays an unusually strong $6284 \AA$ DIB that is found to be normal in fullerene PNe. We also report the detection of new broad and unidentified features centered at $4000 \AA$ and 6525 $\AA$ in DY Cen and Tc 1, respectively. We suggest that the new $4000 \AA$ band seen in DY Cen may be related to the circumstellar proto-fullerenes seen at infrared wavelengths (García-Hernández et al. 2012a). However, the intense $4428 \AA$ DIB (probably also the $6309 \AA$ DIB and the new 6525 $\AA$ band) may be related to the presence of larger fullerenes (e.g., $\mathrm{C}_{80}, \mathrm{C}_{240}, \mathrm{C}_{320}$, and $\mathrm{C}_{540}$ ) and buckyonions (multishell fullerenes such as $\mathrm{C}_{60} @ \mathrm{C}_{240}$ and $\mathrm{C}_{60} @ \mathrm{C}_{240} @ \mathrm{C}_{540}$ ) in the circumstellar envelope of Tc 1 (García-Hernández \& Díaz-Luis 2013).
\end{abstract}

Keywords. Astrochemistry, circumstellar matter, planetary nebulae: general, stars: white dwarfs

\section{Fullerenes in RCB stars and $\mathrm{PNe}$}

Fullerenes (e.g., $\mathrm{C}_{60}, \mathrm{C}_{70}$ ) are very stable molecules that are very important for interstellar/circumstellar chemistry and that may explain many astrophysical phenomena such as the mysterious diffuse interstellar bands (DIBs) and the intense UV bump at $2170 \AA$ (e.g., Iglesias-Groth 2007; Cataldo \& Iglesias-Groth 2009). Fullerenes were discovered in the laboratory (Kroto et al. 1985) and they have been found on Earth and on meteorites. Indeed, the 9577 and $9632 \AA$ DIBs observed in some reddened stars lie near two electronic transitions of $\mathrm{C}_{60}{ }^{+}$(Foing \& Ehrenfreund 1994; see also Berné et al. 2013 for a recent IR detection of the $\mathrm{C}_{60}$ cation). At laboratory, fullerenes are efficiently produced under H-poor conditions (e.g., Kroto et al. 1985) and the so-called R Coronae Borealis (RCB) stars (extremely H-deficient stars) were thus expected to efficiently produce fullerenes (Goeres \& Sedlmayr 1992). In 2010, García-Hernández, Rao \& Lambert looked for $\mathrm{C}_{60}$ in a complete sample of about 30 RCBs by using the Spitzer Space Telescope. We got the unexpected result that $\mathrm{C}_{60}$-like mid-IR features were only detected in those RCBs with some H (García-Hernández et al. 2011a). In particular, $\mathrm{C}_{60}$-like emission features were detected in the least H-deficient RCBs DY Cen and V854 Cen, which also show strong polycyclic aromatic hydrocarbon (PAH) features. Because of the unexpected result in $\mathrm{RCBs}$, then we looked for fullerenes in $\sim 240$ Planetary Nebulae (PNe) by using data from our own Spitzer projects; five clear fullerene detections were found. Meanwhile, 

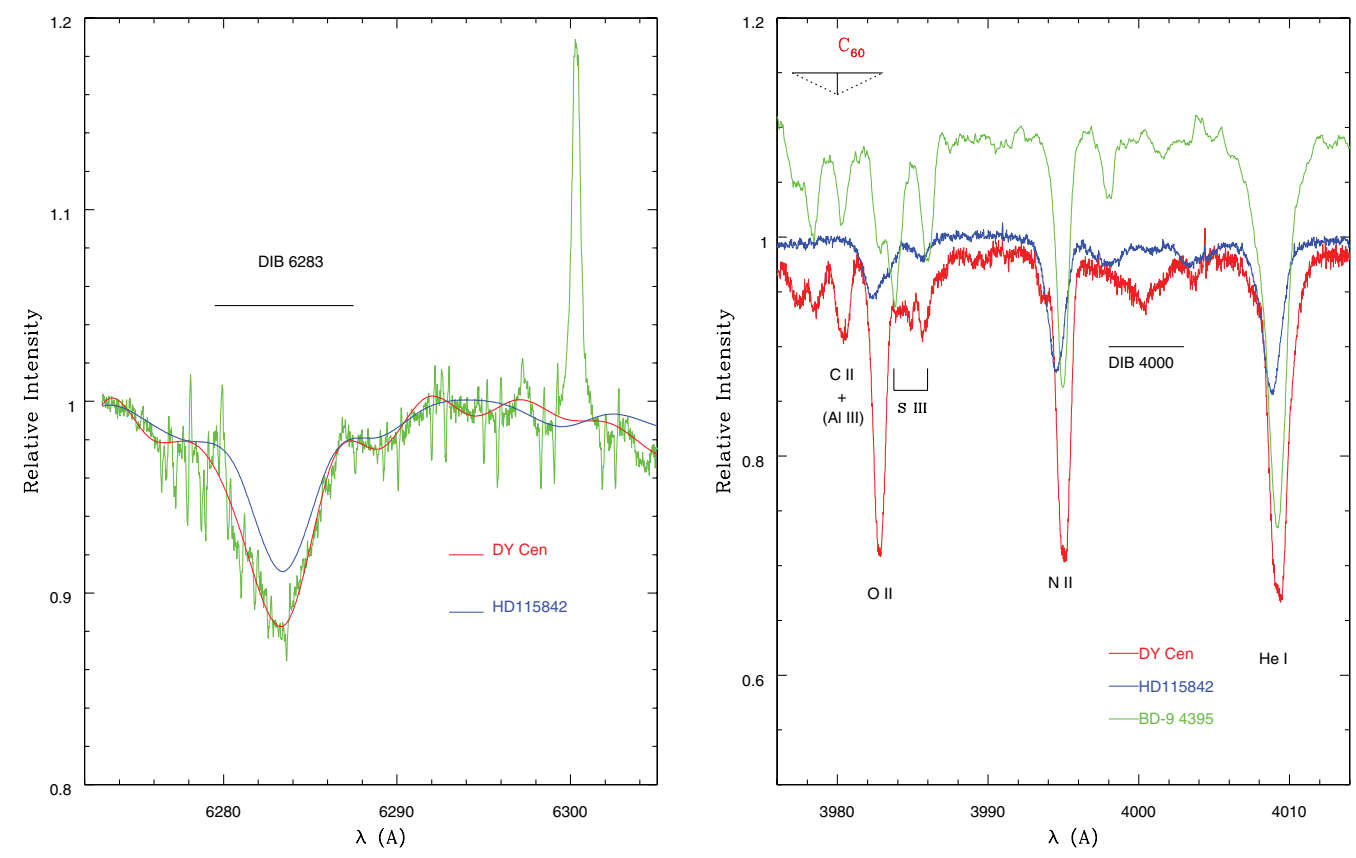

Figure 1. Left panel: Spectral region around the $6284 \AA$ DIB in DY Cen (in red) and HD 115842 (in blue). The telluric line corrected spectrum of both stars are shown by smooth lines (red and blue). The non telluric corrected spectrum in DY Cen is also shown (in green). Right panel: The spectra of DY Cen (in red) and HD 115842 (in blue) around and $4000 \AA$ where the extreme He star $\mathrm{BD}-9^{\circ} 4395$ is also displayed (in green). The expected position and FWHM of the $\mathrm{C}_{60}$ feature at $3980 \AA$ are marked on top of the spectra; there is no evidence (additional absorption) in DY Cen for the presence of this neutral $\mathrm{C}_{60}$ feature. However, there is an additional absorption band at $4000 \AA$ in DY Cen, which is not present in HD 115842 neither in BD $-9^{\circ} 4395$.

Cami et al. (2010) reported the extraordinary discovery of the first IR detection of $\mathrm{C}_{60}$ and $\mathrm{C}_{70}$ fullerenes in the young PN Tc 1. At the same time, García-Hernández et al. (2010) confirmed the surprising results obtained in RCBs, showing that all PNe with fullerenes (including Tc 1) are low-mass C-rich PNe with normal $\mathrm{H}$ abundances. Contrary to the RCB stars, the Spitzer PNe spectra display $\mathrm{C}_{60}$-like features in conjunction with very weak PAHs. This challenged our understanding of the fullerenes formation in space, showing that, contrary to general expectation, fullerenes are efficiently formed in $\mathrm{H}$-rich circumstellar environments only. Furthermore, the detection of fullerenes in RCB stars and PNe suggests that large fullerenes may be formed as decomposition products of hydrogenated amorphous carbon (HAC) dust (García-Hernández et al. 2010, 2011a,b, 2012b; Bernard-Salas et al. 2012; Micelotta et al. 2012).

Thus, fullerenes and related large C-based molecules (e.g., other fullerenes as stable exohedral or endohedral metallo-complexes) might be ubiquitous in the interstellar medium and continue to be serious candidates for the DIB carriers. A detailed analysis of the DIBs towards fullerene-containing - accompanied or not by PAH molecules space environments is very useful to learn about the nature of the DIB carriers. In this context, the recent detections of large fullerene-like molecules in RCBs and $\mathrm{PNe}$ offer a beautiful opportunity for studying the DIB spectrum of sources where fullerenes and fullerene-related molecules are abundant. Here we present for the first time a detailed inspection of the optical spectra of the hot RCB star DY Cen and two fullerene PNe (Tc 1 and M 1-20), which permits us to directly explore the fullerenes - DIB connection. 

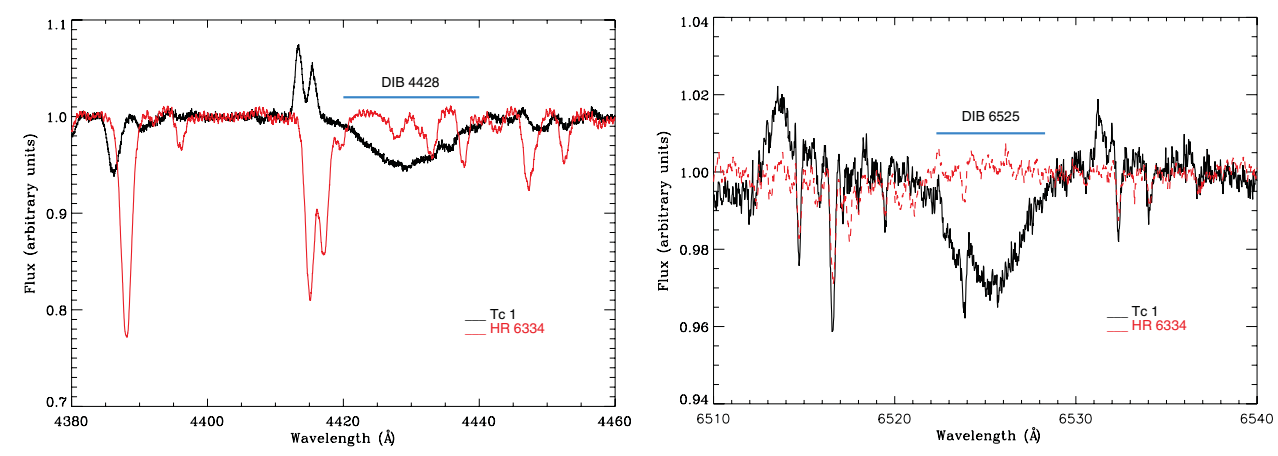

Figure 2. Left panel: Spectra of Tc 1 (in black) and HR 6334 (in red) around the $4428 \AA$ DIB. This DIB is found to be unusually strong in Tc 1 while HR 6334 - with a higher E(B-V) of 0.42 - does not show evidence of its presence. Right panel: Spectral region around the new broad unidentified band at $6525 \AA$ in Tc 1 (in black) and HR 6334 (in red).

\section{Our DIB survey}

We acquired high-resolution $(\mathrm{R} \geqslant 15,000)$ and high signal-to-noise ( $\mathrm{S} / \mathrm{N} \geqslant 200)$ VLTUVES optical ( $\sim 3300-9450 \AA)$ spectra of the RCB star DY Cen and PN Tc 1. The PN M 1-20 was also observed although at lower S/N. Nearby B-type comparison stars were observed on the same dates as the corresponding science objects using the same VLTUVES set-up. The observational details are not repeated here and we refer the reader to García-Hernández et al. (2012a) and García-Hernández \& Díaz-Luis (2013) for the observations of DY Cen and Tc 1 (and M 1-20), respectively. The Spitzer IR spectrum of DY Cen is dominated by PAH-like features with weaker $\mathrm{C}_{60}$-like features (GarcíaHernández et al. 2011a) while Tc 1 displays a $\mathrm{C}_{60}$-dominated IR spectrum with very weak (or absent) PAH bands (Cami et al. 2010; García-Hernández et al. 2010). The goal of our optical observations is to study the characteristics of DIBs in fullerene-containing environments as well as to shed some light about the possible fullerenes - DIB connection.

We find that the classical and well-studied DIBs (e.g., those at 4428, 5780, 5797, 5850, 6196,6379 , and $6614 \AA$ ) towards DY Cen are normal for its reddening. The only exception is the DIB at $6284 \AA$ (possibly also the $7223 \AA$ DIB) (see García-Hernández et al. 2012a for more details). Figure 1 (left panel) shows the region of $6284 \AA$ for DY Cen and the nearby comparison star HD 115824. It is clear that this DIB towards DY Cen is stronger than towards HD 115842, suggesting that the carrier of the $6284 \AA$ DIB (along with 7223 $\AA$ ) is different from the rest of the classical DIBs. Also interesting is that we detect in DY Cen a broad (FWHM $\sim 2 \AA$ ) unidentified feature centered at $\sim 4000 \AA$, which is seen in DY Cen only (Fig. 1; right panel). Note that no DIBs are known at this wavelength (see e.g., Hobbs et al. 2008) and no molecule is known to exhibit a strong electronic transition at $\sim 4000 \AA$. In addition, García-Hernández et al. (2012a) have reported the non-detection of the strongest $\mathrm{C}_{60}$ electronic transitions (e.g., those at $\sim 3760,3980$, and $4024 \AA$ ) in DY Cen (see Fig. 1, right panel); $C_{60}$ IR column density estimates are 1000 times higher than the optical detection limits.

Similarly to DY Cen, we find the strongest DIBs (e.g., those at 5780, 5797, 5850, $6196,6270,6284,6380$, and $6614 \AA$ ) most commonly found in the ISM to be normal in Tc 1 and M 1-20 (see García-Hernández \& Díaz-Luis 2013 for more details). This may suggest that the carriers of the latter well-studied DIBs are not particularly overabundant in fullerene PNe. Surprisingly, the well-studied DIB at $4428 \AA$ as well as the weaker $6309 \AA$ DIB (see e.g., Hobbs et al. 2008) are found to be unusually strong towards Tc 1; the $4428 \AA$ DIB is unusually strong in M 1-20 too. Figure 2 (left panel) compares the 
Table 1. Overview of DIBs in fullerene-containing RCBs and PNe.

\begin{tabular}{|c|c|c|}
\hline DIB & $\mid \begin{array}{c}\text { RCBs } \\
\text { (DY Cen) }\end{array}$ & $\begin{array}{c}\mathbf{P N e} \\
(\mathrm{Tc} 1)\end{array}$ \\
\hline $\mid \begin{array}{ll}4000 & \AA \\
4428 \AA \\
6284 \AA \\
6309 \AA \\
6525 \AA\end{array}$ & $\begin{array}{c}\text { yes } \\
\text { normal } \\
\text { strong } \\
\text { no } \\
\text { no }\end{array}$ & $\begin{array}{c}\text { no } \\
\text { strong } \\
\text { normal } \\
\text { yes } \\
\text { yes }\end{array}$ \\
\hline
\end{tabular}

$4428 \AA \mathrm{DIB}$ in Tc 1 with that in the nearby comparison star HR 6334. Adopting a Lorentzian profile for the $4428 \AA \mathrm{DIB}$, we obtain an EQW of $860 \mathrm{m \AA}$, which is at least a factor of two greater than expected from the reddening in Tc 1. An unidentified broad (FWHM $\sim 5 \AA$ ) feature at $6525 \AA$ is also detected in Tc $1 \uparrow$. Figure 2 (right panel) displays the unidentified $6525 \AA$ band in the Tc 1 spectrum in comparison with the star HR 6334 . Again, the most intense optical bands of neutral $\mathrm{C}_{60}$ are lacking in the Tc 1 spectrum; although this could be explained by the low $\mathrm{C}_{60}$ column density estimated from the $\mathrm{C}_{60}$ IR features if the neutral $\mathrm{C}_{60}$ emission peaks far away from the central star (GarcíaHernández \& Díaz-Luis 2013). Finally, the DY Cen's unidentified $4000 \AA$ band is not seen in Tc 1.

\section{Diffuse interstellar bands in (proto-) fullerene-rich environments}

Table 1 compares the DIBs seen in the RCB star DY Cen with those in the fullerene PN Tc 1. This table naturally prompts the question of why DIBs are so different in the fullerene-containing environments around RCB stars and PNe?

Based on their laboratory spectroscopy of HAC nanoparticles, Duley \& Hu (2012) propose that the $\mathrm{C}_{60}$ IR features seen in sources with PAH-like dominated IR spectra such as DY Cen are attributable to proto-fullerenes or fullerene precursors rather than to $\mathrm{C}_{60}$. Our non-detection of neutral $\mathrm{C}_{60}$ in the DY Cen optical spectrum may support the Duley \& Hu (2012) HAC laboratory results. Thus, the new $4000 \AA$ band seen in DY Cen may be related to the circumstellar proto-fullerenes; perhaps an organic compound containing pentagonal rings. These pentagonal carbon rings are usually present in HAC nanoparticles and nanotubes, suggesting that they may be intimately related with the formation process of fullerenes. In addition, the $4428 \AA$ DIB has been linked to fullerenes bigger than $\mathrm{C}_{60}$ and/or buckyonions such as $\mathrm{C}_{60} @ \mathrm{C}_{240}$ and $\mathrm{C}_{60} @ \mathrm{C}_{240} @ \mathrm{C}_{540}$ (IglesiasGroth 2007). Our findings in DY Cen would be consistent with fullerenes and fullerenescontaining molecules not being especially overabundant towards DY Cen.

On the other hand, the non detection of neutral $\mathrm{C}_{60}$ in the Tc 1 optical spectrum is intriguing. Tc 1 - with no or very weak PAHs - is expected to be rich in $\mathrm{C}_{60}(\mathrm{Du}-$ ley \& $\mathrm{Hu}$ 2012). An exotic explanation may be that larger fullerenes or more complex fullerene-based molecules are present. This is suggested by the unusually strong $4428 \AA$ DIB. Photo-absorption theoretical models of large fullerenes $\left(\mathrm{C}_{80}, \mathrm{C}_{240}, \mathrm{C}_{540}\right)$ and buckyonions $\left(\mathrm{C}_{60} @ \mathrm{C}_{240}, \mathrm{C}_{60} @ \mathrm{C}_{240} @ \mathrm{C}_{540}\right)$ (Iglesias-Groth 2007) display strong transitions around $4428 \AA$. In this framework, the $4428 \AA$ DIB may be explained by the transitions (superposition) of fullerenes bigger than $\mathrm{C}_{60}$ and multishell fullerenes (buckyonions)

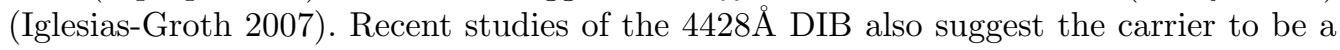
resistant, large and compact neutral molecule (van Loon et al. 2013).

$\dagger$ A few other unidentified bands and/or unusually strong DIBs seem to be present in Tc 1 (see Manchado et al. these proceedings). 


\section{Concluding remarks}

The IR detection of $\mathrm{C}_{60}$ in RCB stars and PNe offers the opportunity of studying DIBs in environments where fullerenes are abundant. We have shown here that DIBs in RCB stars and PNe are remarkably different. The new $4000 \AA$ band detected in the RCB star DY Cen is suggested to be related with proto-fullerenes or fullerene-precursors (García-

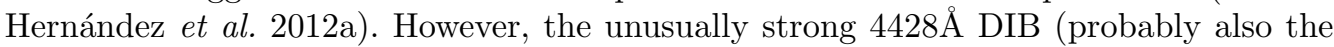
weaker $6309 \AA$ DIB and the unidentified $6525 \AA$ band) in PNe is suggested to be related with the presence of large fullerenes and buckyonions (García-Hernández \& Díaz-Luis 2013) as previously pointed out by Iglesias-Groth (2007) from theoretical considerations.

At present, the HAC's photochemical processing seems to be the most promising $\mathrm{C}_{60}$ formation route; at least in the complex circumstellar envelopes of RCB stars and PNe (see e.g., García-Hernández et al. 2012b; and references therein) but see also Berné \& Tielens (2012). Larger fullerenes and fullerene-based molecules may form from pre-existing $\mathrm{C}_{60}$ molecules (e.g., Dunk et al. 2012), opening the possibility of forming a rich family of fullerene-related molecules such as buckyonions, metallofullerenes, and fullerene adducts. These complex fullerene-based molecules may emit through the same IR vibrational modes (e.g., as isolated $\mathrm{C}_{60}$ and $\mathrm{C}_{70}$ ), being indistinguishable from $\mathrm{C}_{60}$ (and $\mathrm{C}_{70}$ ) on the basis of IR spectra alone. In particular, fullerenes and PAHs may be mixed in the circumstellar envelopes of fullerene PNe (e.g., M 1-20) and fullerene/PAH adducts may form via Dies-Alder cycloaddition reactions (García-Hernández et al. 2013). Indeed, very recent laboratory work demonstrates that fullerene/PAH adducts - such as $\mathrm{C}_{60}$ /anthracene Diels-Alder adducts - display mid-IR features strikingly coincident with those from $\mathrm{C}_{60}$ and $\mathrm{C}_{70}$ (García-Hernández et al. 2013).

\section{Acknowledgements}

D.A.G.H. acknowledges support provided by the Spanish Ministry of Economy and Competitiveness under grant AYA-2011-29060.

\section{References}

Bernard-Salas, J., Cami, J., Peeters, E., et al. 2012, ApJ, 757, 41

Berné, O. \& Tielens, A. G. G. M. 2012, PNAS, 109, 401

Berné, O., Mulas, G., \& Joblin, C. 2013, A\&SA, 550, L4

Cami, J., Bernard-Salas, J., Peeters, E., \& Malek, S. E. 2010, Science, 329, 1180

Cataldo, F. \& Iglesias-Groth, S. 2009, MNRAS, 400, 291

Dunk, P. W., Kaiser, N. K., Hendrickson, C. L., et al. 2012, Nature Commun., 3, 855

Duley, W. W. \& Hu, A. 2012, ApJ, 745, L11

Foing, B. H. \& Ehrenfreund, P. 1994, Nature, 369, 296

García-Hernández, D. A., Cataldo, F., \& Manchado, A. 2013, MNRAS, 434, 415

García-Hernández, D. A. \& Díaz-Luis, J. J. 2013, A\&\&A, 550, L6

García-Hernández, D. A., Iglesias-Groth, S, Acosta-Pulido, J. A., et al. 2011b, ApJ, 737, L30

García-Hernández, D. A., Manchado, A., García-Lario, P., et al. 2010, ApJ, 724, L39

García-Hernández, D. A., Rao, N. K., \& Lambert, D. L. 2011a, ApJ, 729, 126

-. 2012a, ApJ, 759, L21

García-Hernández, D. A., Villaver, E., García-Lario, P., et al. 2012b, ApJ, 760, 107

Goeres, A. \& Sedlmayr, E. 1992, A\&SA, 265, 216

Hobbs, L. M., York, D. G., Snow, T. P., et al. 2008, ApJ, 680, 1256

Iglesias-Groth, S. 2007, ApJ, 661, L167

Kroto, H. W., Heath, J. R., Obrien, S. C., et al. 1985, Nature, 318, 162

Micelotta, E. R., Jones, A. P., Cami, J., et al. 2012, ApJ, 761, 35

Van Loon, J. Th., Bailey, M., Tatton, B. L., et al. 2013, A\&A, 550, A108 